\title{
Acute transverse myelitis in Lyme neuroborreliosis
}

\author{
S. Bigi $\cdot$ C. Aebi $\cdot$ C. Nauer $\cdot$ S. Bigler $\cdot$ \\ M. Steinlin
}

Received: 14 January 2010/Accepted: 3 May 2010/Published online: 27 May 2010

(C) Urban \& Vogel 2010

\begin{abstract}
Introduction Acute transverse myelitis (ATM) is a rare disorder (1-8 new cases per million of population per year), with $20 \%$ of all cases occurring in patients younger than 18 years of age. Diagnosis requires clinical symptoms and evidence of inflammation within the spinal cord (cerebrospinal fluid and/or magnetic resonance imaging). ATM due to neuroborreliosis typically presents with impressive clinical manifestations.

Case presentation Here we present a case of Lyme neuroborreliosis-associated ATM with severe MRI and CSF findings, but surprisingly few clinical manifestations and late conversion of the immunoglobulin G CSF/blood index of Borrelia burgdorferi sensu lato.

Conclusion Clinical symptoms and signs of neuroborrelial ATM may be minimal, even in cases with severe involvement of the spine, as shown by imaging studies. The CSF/blood index can be negative in the early stages and does not exclude Lyme neuroborreliosis; if there is
\end{abstract}

S. Bigi $(\bowtie) \cdot$ M. Steinlin

Neuropaediatrics, Department of Paediatrics,

University Children's Hospital, Inselspital,

Bern, Switzerland

e-mail: Sandra.bigi@insel.ch

C. Aebi

Paediatric Infectious Diseases, Department of Paediatrics,

University Children's Hospital, Inselspital, Bern, Switzerland

C. Nauer

Neuroradiology, Department of Radiology, University Hospital, Inselspital, Bern, Switzerland

\section{S. Bigler}

Institute of Infectious Diseases, University Hospital,

Inselspital, Bern, Switzerland strong clinical suspicion of Lyme neuroborreliosis, appropriate treatment should be started and the CSF/blood index repeated to confirm the diagnosis.

Keywords Lyme $\cdot$ Neuroborreliosis $\cdot$ Transverse myelitis

\section{Introduction}

Acute transverse myelitis (ATM) is an inflammatory myelopathy with an incidence of $1-8$ cases per million of population per year. Of all affected patients, $20 \%$ are younger than 18 years of age [1]. Diagnosis is based on clinical symptoms, cerebral spinal fluid (CSF) findings and spinal neuroimaging results. In addition to the inflammatory myelopathies, demyelination, infection (e.g. polymyelitic enteroviral infection) and other inflammatory disorders (i.e. systemic lupus erythematodes, neurosarcoidosis) must be considered in the differential diagnosis $[2,3]$. In adults, multiple sclerosis and neuromyelitis optica together with infectious/parainfectious aetiologies are the most frequent causes of ATM [4], while in children the aetiology is mainly postinfectious [5]. Clinical features consist of sensory disturbances in most of the patients, followed by weakness and-especially in childhood-urinary tract dysfunction. Children suffer from more severe clinical impairment then adults. In the series of Pidcock et al., $89 \%$ of the paediatric patients were bed- or wheelchair bound or required assisted ventilation, whereas the majority of adults showed only mild impairment [1, 6]. Outcome is generally favourable. Chronic bladder dysfunction has been shown to be the main sequelae [1].

ATM due to Lyme neuroborreliosis has rarely been reported. However, those cases that have been documented, the majority of patients presented with impressive clinical 
Fig. 1 Left T2-weighted sagittal scan of the cervicothoracic spine. There is an extensive centromedullary hyperintensity (arrow) and swelling as an expression of the spinal cord edema. Right T2-weighted axial scan at the level of the cervical spine. The edema involving the gray and white matter is discernible. The hyperintensity of the gray matter (arrow) is particularly prominent

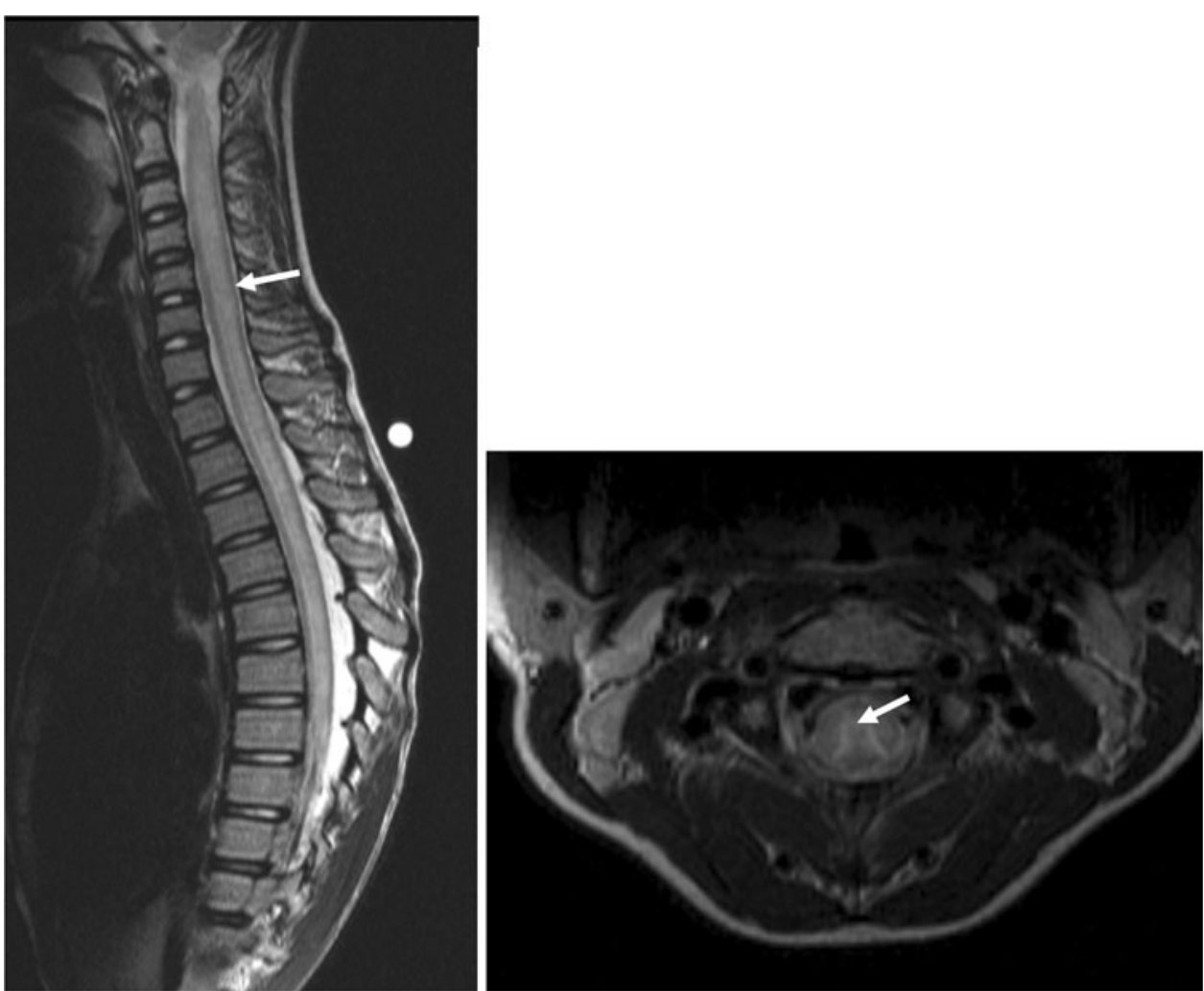

symptoms [7-9]. Here, we report a case of ATM due to Lyme neuroborreliosis, which-despite severe neuroimaging (magnetic resonance imagining, MRI) and CSF findings-was characterized by surprisingly few clinical signs.

\section{Case report}

A 12.5-year-old boy presented to our emergency department in November with a $>4$-week history of increasing frontal headache that got worse in the evening. He noted intermittent subfebrile temperatures. Additional complaints were fatigue, diffuse non-radicular back/neck pain and nausea. There was no vomiting. In this period, a weight loss of $2-3 \mathrm{~kg}$ was documented. Three weeks before admission he had been immunized (dTap = diphtheria, tetanus, acellular pertussis) according to the recommended Swiss vaccination schedule. His personal history is unremarkable. There was no tick bite reported, and no an erythema migrans.

On admission, the general physical condition of the patient was slightly lower than would normally be expected, he was afrebrile, and the results of a general clinical examination were normal. His neurological examination was remarkable only for nuchal rigidity. In particular, there was no bladder dysfunction, and there was no sensory disturbance or weakness.

Laboratory investigations on admission included a normal complete blood cell count, inflammatory markers (C-reactive protein), liver enzymes and kidney function parameters.

Because of the clinical constellation (neck stiffness in an afebrile patient), MRI of the brain was performed to exclude a haemorrhagic or space-occupying lesion. The brain scan was normal, but there were signal alterations (T2w hyperintensities) within the medulla down to cervical spine. A consecutive MRI of the spinal cord was added to the tests, which revealed a longitudinal signal alteration (T2w hyperintensities) from the pons to the conus, accentuated centromedullarily with a patchy enhancement (Fig. 1). The myelon was swollen, especially in the cervical region. There was no tumor or cerebral or meningeal enhancement on the MRI scan.

There was marked CSF pleocytosis (787cells/ $\mu \mathrm{l} ; 734$ mononuclear cells), elevated protein $(6.24 \mathrm{~g} / \mathrm{l})$ and decreased glucose $(1.79 \mathrm{mmol} / \mathrm{l})$, with a glucose $\mathrm{CSF} /$ plasma ratio of 0.28 . Microbiological investigation revealed increased serum immunoglobulin $\mathrm{G}$ ( $\mathrm{IgG}$ ) and IgM for Epstein-Barr virus (EBV) (EBV viral capsid antigen- $\operatorname{IgM}$ and - $\mathrm{IgG}$, anti-early-antigen $\operatorname{IgG}$ p54) and Borrelia burgdorferi sensu lato. Serological testing for 
Fig. 2 Left T2-weighted sagittal scan of the cervicothoracic spine. The centromedullary edema is regressive but still visible. Right T2-weighted axial scan at the level of the cervical spine. Central hyperintensity with accentuation in the central grey matter was present, but was less marked than in the initial scan

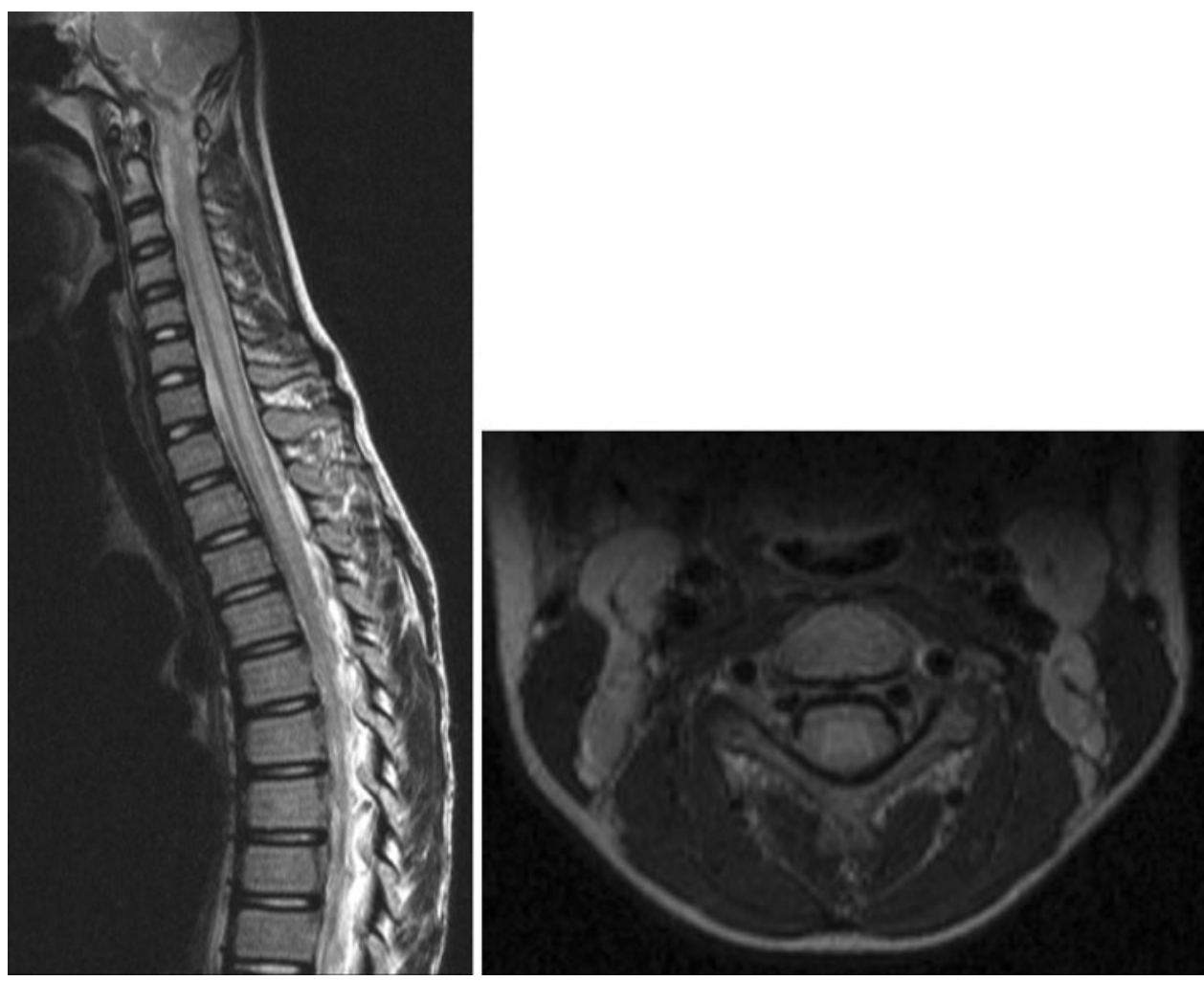

B. burgdorferi was performed using a recomWell enzymelinked immunosorbent assay (ELISA; IgM and IgG; Mikrogen, Neuried, Germany) and confirmed by recomBlot (Mikrogen; IgM and IgG). The Western blot revealed that the patient had borrelia-specific positive $\operatorname{IgM}$ and $\operatorname{IgG}$ bands that were distributed as follows: anti-OspC IgM (B. burgdorferi sensu lato); anti-p41 flagellin IgM; anti-p41 intern $\operatorname{IgM}$ (B. afzelii); anti-VisE IgG (B. afzelii); anti-p41 flagellin IgG; anti-p41-intern IgG (B. garinii); anti-p41intern IgG (B. afzelii). The EBV DNA PCR of the CSF and the CSF/blood index for B. burgdorferi sensu lato $\operatorname{IgM}$ and IgG were negative. The CSF/blood index was performed using the IDEIA test (Oxoid, Cambridge, UK) for Lyme neuroborreliosis. The results of the CSF PCR for herpes simplex virus (HSV) and enteroviruses were negative.

With the suspicion of Lyme neuroborreliosis, ceftriaxone therapy for 14 days was started. Antiviral therapy with aciclovir was added at the beginning of the therapeutic regimen and stopped when the negative HSV PCR result became available. Steroids had not been given. The patient recovered almost completely within the first $24 \mathrm{~h}$ of therapy, with only residual mild neck stiffness that persisted for 1 week. Eight days after the initiation of therapy, a followup MRI of the spinal cord showed improvement with reduced swelling and less signal alteration (Fig. 2).

Three weeks after admission, an outpatient control showed a completely normal clinical examination. In the follow-up lumbar puncture performed at this time, 27 cells $/ \mu 1$ were detected (all mononuclear). Protein and glucose (including CSF/plasma ratio) were normal. Examination of the oligoclonal IgG bands in the CSF/serum revealed an intrathecal IgG production. The IgG CSF/blood index for B. burgdorferi sensu lato was $41.73(<0.3)$, and the EBV DNA PCR of the CSF remained negative.

\section{Discussion}

Lyme neuroborreliosis as a cause of ATM is known, but reports are limited to single cases, both in children and adults. The usual manifestations are severe and often include dramatic signs of motor weakness and bladder dysfunction [7]. In discussing our case, we wish to emphasize two aspects which - to the best of our knowledge-have not been reported to date: the striking discrepancy between the severe imaging manifestations and the minimal clinical signs and symptoms, and the prominent MRI findings involving most of the medulla spinalis in a patient with transverse myelitis of borrelial origin. In pediatric Lyme neuroborreliosis, CNS symptoms are rare-in contrast to symptoms of the peripheral nervous system. Children with CNS symptoms may present with acute myelitis. In all such cases reported so far, the children suffered from acute symptoms due to their myelitis, such as 
pareses, urinary tract dysfunction and sensory disturbances. All of these symptoms and signs were lacking in our patient, possibly because of the early diagnosis and treatment [10]. The description of an extension to spine involvement in a transverse myelitis of borrelial origin in children is limited to single case reports. In these case reports, the involvement of the spine is limited and mostly cervically $[7,11]$. At the moment of first lumbar puncture, our patient had a symptom duration of $<6$ weeks, which explains the first negative CSF/blood index, as delayed intrathecal antibody synthesis in Lyme neuroborreliosis (positive CSF/blood index) is well known in patients with a symptom duration of $<6$ weeks. Therefore, the positive $\mathrm{CSF} / \mathrm{blood}$ index in the follow-up lumbar puncture confirms the assumed diagnosis of Lyme neuroborreliosis, and early detection and treatment may have prevented further progression of the disease. [12-14] In the literature, EBVin addition to many other occasionally incriminated viruses-is a rare but recognized agent causing ATM [5, 15]. Serologically, our patient had a co-infection with EBV at the time of his CNS manifestation, even though there was no classic mononucleosis (tonsillitis, lymphadenopathy, hepatosplenomegaly) and the EBV DNA PCR of the CFS remained negative over time. We conclude that the ATM was related to Lyme neuroborreliosis and not to the EBV infection. However, this patient most likely has had a recent infection with $\mathrm{EBV}$, which may or may not have paved the way for progression of early, local borreliosis to Lyme neuroborreliosis.

Single cases of ATM after vaccination have been reported, but in these, in contrast to our case, there were no other aetiological findings. Pidcock et al. reported that $28 \%$ of the reported children with ATM received immunization or "allergy shots" within 30 days prior to onset, but they could not demonstrate a potential causal link between vaccination and ATM. In our case, the onset of symptoms 1 week prior to vaccination renders a vaccine-related adverse effect unlikely, but the possibility of vaccination supporting the development of Lyme neuroborreliosis because of an already present infection cannot be entirely ruled out or resolved.

In conclusion, we report the case of a patient with ATM caused by Lyme neuroborreliosis with surprisingly few clinical signs - in contrast to the severe MRI and CSF findings. If there is strong clinical suspicion of Lyme neuroborreliosis - irrespective of a history of a recent tick bite or erythema migrans-appropriate treatment should be started, and the CSF/blood index should be repeated to confirm the diagnosis if the initial results are negative.

Conflict of interest statement None.

\section{References}

1. Pidcock FS, Krishnan C, Crawfor TO, Salorio CF, Trovato. M, Kerr DA. Acute transverse myelitis in childhood: center-based analysis of 47 cases. Neurology. 2007;68:1474-80.

2. Defresne P, Hollenberg H, Husson B, et al. Acute transverse myelitis in children: clinical course and prognostic factors. J Child Neurol. 2003;18:401-6.

3. Jacob A, Weinshenker BG. An approach to the diagnosis of acute transverse myelitis. Semin Neurol. 2008;28:105-20.

4. de Seze J, Lanctin C, Lebrun C, et al. Idiopathic acute transverse myelitis: application of the recent diagnostic criteria. Neurology. 2005;65:1950-3.

5. Knebusch M, Strassburg HM, Reiners K. Acute transverse myelitis in childhood: nine cases and review of the literature. Dev Med Child Neurol. 1998;40:631-9.

6. Sellner J, Lüthi N, Schüpbach WMM, et al. Diagnostic workup of patients with acute transverse myelitis: spectrum of clinical presentation, neuroimaging and laboratory findings. Spinal Cord. 2009;47:312-7.

7. Huisman TA, Wohlrab G, Nadal D, Boltshauser E, Martin E. Unusual presentations of neuroborreliosis (Lyme disease) in childhood. J Comput Assist Tomogr. 1999;23:39-42.

8. Olivares JP, Pallas F, Cecaldi M, Viton JM, et al. Lyme disease presenting as isolated acute urinary retention caused by transverse myelitis: an electrophysiological and urodynamical study. Arch Phys Med Rehabil. 1995;76:1171-2.

9. Rousseau JJ, Lust C, Zangerlie PF, Bigaigon G. Acute transverse myelitis as presenting neurological feature of Lyme disease. Lancet. 1986;2:1222-3.

10. Mygland A, Ljøstad U, Fingerle V, Rupprecht T, Schmutzhard E, et al. EFNS guidelines on the diagnosis and management of European Lyme neuroborreliosis. Eur J Neurol. 2010; 17:8-16

11. Meurs L, Labeye, D., Declercq, I., Pieret, F., and Gille M. Acute transverse myelitis as a main manifestation of early stage II neuroborreliosis in two patients. Eur Neurol. 2004;52:186-8.

12. Christen HJ, Hanefeld, F, Eiffert, H, Thomssen, R. Epidemiology and clinical manifestations of Lyme borreliosis in childhood. A prospective multicentre study with special regard to neuroborreliosis. Acta Paediatr Suppl. 1993;386:1-75.

13. Blanc F, Jualhac B, Fleury M, et al. Relevance of the antibody index to diagnose Lyme neuroborreliosis among seropositive patients. Neurology. 2007;69:953-8.

14. Ljostad U, Skarpaas T, Mygland A. Clinical usefulness of intrathecal antibody testing in acute Lyme neuroborreliosis. Eur J Neurol. 2007;14:873-6.

15. Doja A, Bitnun A., Jones EL, et al. Pediatric Epstein-Barr virusassociated encephalitis: 10-year review. J Child Neurol. 2006;21:385-91. 